WellBeing International

WBI Studies Repository

1985

\title{
T-61 Use in the Euthanasia of Domestic Animals: A Survey
}

Andrew N. Rowan

Tufts University

Follow this and additional works at: https://www.wellbeingintlstudiesrepository.org/acwp_vsm

Part of the Animal Studies Commons, Other Animal Sciences Commons, and the Veterinary Toxicology and Pharmacology Commons

\section{Recommended Citation}

Rowan, A.N. (1985). T-61 use in the euthanasia of domestic animals: A survey. In M.W. Fox \& L.D. Mickley (Eds.), Advances in animal welfare science 1985/86 (pp. 79-86). Washington, DC: The Humane Society of the United States.

This material is brought to you for free and open access by WellBeing International. It has been accepted for inclusion by an authorized administrator of the WBI Studies Repository. For more information, please contact wbisr-info@wellbeingintl.org.

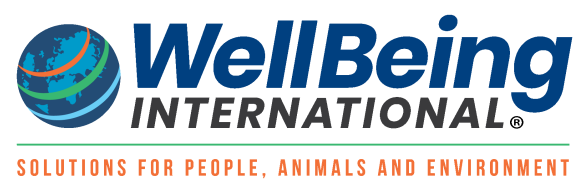




\title{
T-61 USE IN THE EUTHANASIA OF DOMESTIC ANIMALS: A SURVEY
}

\author{
Andrew N. Rowan \\ Department of Environmental Studies \\ Thefts University \\ School of Veterinary Medicine \\ 203 Harrison Avenue \\ Boston, MA 02111
}

\section{Introduction}

A variety of techniques have been proposed and employed for the killing of domestic animals but relatively few have survived as suitable agents for euthanasia - namely, the induction of painless, suffering-free death. Some agents, such as strychnine, curariform agents, or potassium salts cause suffering while others have other disadvantages. Today, dogs and cats are commonly euthanatized with sodium pentobarbital or with T-61 which is a mixture of a central nervous system narcotic, a paralytic agent, and a local anesthetic. The use of T-61 was first reported in the United States in 1963 (Quin 1963). The substance gradually became more popular because it was not a DEA-controlled substance and therefore practitioners did not have to deal with the stringent reporting requirements needed for the barbiturates. However, the presence of a paralytic agent in the T-61 mixture, continuing anecdotal reports of bad reactions when using T-61, and the relatively complicated protocol recommended for its administration have resulted in repeated questions being raised about the appropriateness of T-61 as a euthanasia agent.

\section{History of T-61 Use and Evaluation}

The first reported use of T-61 for the killing of dogs and cats came from West Germany where Eikmeier (1961) concluded from experience on $350 \mathrm{dogs}$ and 300 cats that the material was suitable for small 
animal euthanasia. According to Carding (1977), T-61 use then spread in Europe, in part because it was cheaper than commercial solutions of barbiturate. In America, T-61 use spread more slowly. For example, the 1972 AVMA Panel on Euthanasia did not mention the agent in its report (AVMA 1972), although it was included in the 1978 revision (AVMA 1978).

Although there was some unpublished electroencephalogram data on T-61 effects in the rats - the EEG became isoelectric in four seconds (Carding 1977) -no studies on the dog were reported until 1978 when the relative effectiveness of T-61 and sodium pentobarbital were compared (Lumb et al. 1978). Nine dogs were injected with T-61 and twelve with sodium pentobarbital under carefully controlled conditions including the recording of EEGs and ECGs. The response of the animals to T-61 and sodium pentobarbital was similar except for three dogs receiving barbiturate in which cardiac output resumed. However, as Reilly (1978) noted, the dose of barbiturate used was close to the minimum lethal dose and the sodium pentobarbital solution was one-half to onethird the strength (at $130 \mathrm{mg} / \mathrm{ml}$ ) of barbiturate solutions commonly sold for animal euthanasia today (Lumb and Moreland 1982).

\section{The Survey}

In view of the continuing questions surrounding the use of T-61 for dog and cat euthanasia, it was decided to survey the reported experience of veterinarians with the agent. Four hundred and twenty-three questionnaires were distributed to veterinarians in Massachusetts. Two hundred and thirty-four completed forms were received. This represents a 55.3 percent response rate, which is excellent.

Over 90 percent of the respondents indicated some experience with T-61 but the majority used sodium pentobarbital for routine euthanasia (table 1). Half of the respondents said they did not use T-61, usually because of one or more bad experiences with the drug. Of the rest, 27.8 percent felt the drug was a satisfactory euthanasia agent (several felt it was excellent) while 21.4 percent had no strong opinion either way. Table 2 provides a more detailed breakdown of the opinions.

Table 1. Summary of sample responses.

\begin{tabular}{llcc}
\hline & & Number & \% of Total \\
Routinely use Na Pent & & 162 & 69.2 \\
Routinely use T-61 & & 72 & 30.8 \\
\cline { 2 - 4 } Attitude to T-61 & Pro & 65 & 27.8 \\
& Con (cost) & 14 & 6.0 \\
& Con (bad reactions) & 103 & 44.0 \\
& Neutral/No opinion & 52 & 22.2
\end{tabular}


Table 2. Opinions on T-61 use

\begin{tabular}{|c|c|c|c|c|c|c|}
\hline & \multicolumn{2}{|c|}{ Na Pent Users } & \multicolumn{2}{|c|}{ T-61 Users } & \multicolumn{2}{|c|}{ Total } \\
\hline & No. & $\%$ & No. & $\%$ & No. & $\%$ \\
\hline No stated opinion & 22 & 13.6 & 1 & 1.4 & 23 & 9.8 \\
\hline T-61 too expensive & 14 & 8.6 & - & - & 14 & 6.0 \\
\hline Neutral on T-61 use & 27 & 16.7 & 2 & 2.8 & 29 & 12.4 \\
\hline $\begin{array}{l}\text { T- } 61 \text { causes too many } \\
\text { bad reactions }\end{array}$ & 99 & 61.1 & 4 & 5.6 & 103 & 44.0 \\
\hline Satisfied with or preferT-61 & - & - & 65 & 90.2 & 65 & 27.8 \\
\hline TOTALS & 162 & 100 & 72 & 100 & 234 & 100 \\
\hline
\end{tabular}

There were a number of comments on some of the perceived problems with T-61. Both users and non-users commented that the heart takes a long time to stop beating. Some talked of the heart continuing for as long as five minutes or more after brain death had apparently occurred. One respondent noted that, in 90 percent of cases, the heart continued beating for several minutes. Another feature of T-61 euthanasia that was mentioned by both groups was the presence of muscle tremors and excitation. T-61 users did not make very much of this and indicated that tremors occurred in approximately 10 percent of cases. However, barbiturate users who did not like the drug spoke of excitation, including convulsions, crying and agonal thrashing, in 20 percent or more of cases.

Of those opposed to the use of T-61, one commented that, while the drug does kill the animal, it is not euthanasia. Another argued that T-61's relatively narrow procedural tolerance, relatively high incidence of adverse reactions, and demonstrated severe excitement reactions in large animals make the drug a liability. A third stated that, while the esthetics of barbiturate euthanasia are not always good, they are usually better than those associated with T-61 euthanasia. On the other side, one person commented that, after fifty years of experience, T-61 was by far the best euthanasia agent or method that has been made available. Another noted that T- 61 is an effective euthanasia agent although it would be reassuring to know what it is actually doing in the animal.

There were a number of contradictory comments on specific aspects of T-61 use. Several respondents noted that they had no problems with T-61 because they only used the drug for equine or large animal euthanasia. Others said that the drug was definitely not satisfactory 
for equine euthanasia. Some noted that extra-venous injection of T-61 was very painful and ineffective while others said that intracardiac, intrathoracic, or intraperitoneal injections were effective and did not cause pain. Several respondents argued that one had to use double or triple the recommended dose and there was a wide variation on the question of whether or not T-61 should be administered according to the manufacturer's instructions - namely, two-thirds of the dose should be administered slowly (one milliliter $/ 5$ seconds) and the remainder injected rapidly. Table 3 indicates the range of opinions on this matter. In one response, it was noted that a double dose of T-61 injected very rapidly was satisfactory while small doses injected slowly were not.

Table 3. Opinions on importance of injecting first two-thirds of T-61 dose slowly.

\begin{tabular}{|c|c|c|c|c|c|c|}
\hline & \multicolumn{2}{|c|}{ T-61 Users } & \multicolumn{2}{|c|}{ Na Pent Users* } & \multicolumn{2}{|c|}{ All } \\
\hline & No. & $\%$ & No. & $\%$ & No. & $\%$ \\
\hline Very Important & 26 & 36.1 & 13 & 46.4 & 39 & 39 \\
\hline Moderately Important & 20 & 27.8 & 9 & 32.1 & 29 & 29 \\
\hline Not Important & 26 & 36.1 & 6 & 21.4 & 32 & 32 \\
\hline TOTALS & 72 & 100 & 28 & 100 & 100 & 100 \\
\hline
\end{tabular}

*Many of those (numbering 134 respondents) who were not using T-61 did not complete the section of the questionnaire dealing with the rate of injection.

The questionnaire results also demonstrated a wide range of opinion on the cost issue. T-61 users were evenly divided on whether the agent was more expensive or less expensive to use than other euthanasia products. Barbiturate users who responded on this question indicated that T-61 was more expensive. According to one survey of suppliers, T-61 is considerably more expensive than Fatal-Plus or Euthanasia-6 but about the same price as Beuthanasia-D (Barocio 1983). This is confirmed by Lumb and Moreland (1982) who note that the cost per pound to euthanize an animal with T-61 or Beuthanasia is about three to four times that of the simpler pentobarbital products.

During analysis of the questionnaires, it often seemed as though respondents were reporting on two different drugs, so contradictory were some of the responses and comments. Part of the reason for this may well be due to the fact that T-61 administration does have narrow procedural tolerances and slight differences in technique may produce large differences in reported responses. Some of the reports appear to contradict the published results of Lumb et al. (1978) who noted that the ECG activity lasted longer in the barbiturate-injected dogs than in those receiving T-61. There were a number of reports of the heartbeat 
continuing for up to a minute or more by respondents who had used T-61 and some uneasiness was expressed over this.

While there are some problems with the survey (self-reporting is not as reliable as recorded observations), and the questionnaire did not identify precisely how much experience individuals had had with T-61 (it is possible that some comments were based on very limited experience), it is possible to draw some conclusions. For example, it is clear that T-61 is not always used in the clinic according to the manufacturer's recommendations. It is also clear that there are many questions about T-61 that still need to be resolved. For example, what is the clinical incidence of muscle tremors and/or convulsions and what causes them? Is the prolonged duration of the heartbeat after the injection a problem that needs to be addressed? What is the reason for contradictory reports of T-61 euthanasia in large animals? This survey cannot answer such questions but merely adds to the anecdotal data indicating that the continuing use of T-61 as a euthanasia agent needs to be subjected to close and critical review. 


\section{References}

AVMA. 1972. Report of the AVMA Panel on Euthanasia. J. Am. Vet. Med. Assoc. 160:761-72

AVMA. 1978. Report of the AVMA Panel on Euthanasia. J. Am. Vet. Med. Assoc. 173:59-72

Barocio, L.D. 1983. Review of literature on use of T-61 as a euthanasic agent. Int. J. Study Animal Problems. 4:336-42

Carding, T. 1977. Euthanasia of dogs and cats: An analysis of experience and current knowledge with recommendations for research. Animal Reg. Study. 1:5-21

Eikmeier, M. 1962. Experiences with a new preparation for painless destruction of small animals (T-61). Die Blauen Hefte Tierarztl. 5:22-23

Lumb, W.V. and Moreland, A.F. 1982. Chemical methods for euthanasia. Lab Animal. 11(4):29-35

Lumb, W.V., Doshi, K., and Scott, J.R. 1978. A comparative study of T-61 and pentobarbital for euthanasia of dogs. J. Am. Vet. Med. Assoc. 172:149-52

Quin, A.H. 1963. Observations on a new euthanasia agent for small animals. Vet. Med. 58:494-5

Reilly, L.L. 1978. Euthanasia of dogs. J. Am. Vet. Med. Assoc. 172:984 


\section{Editor's Commentary}

Euthanasia means a humane death. For euthanasia to be humane, therefore, it must induce unconsciousness, not arrest respiration before unconsciousness occurs, and bring about the onset of death swiftly and consistently.

T-61 cannot be regarded as a suitable euthanasia agent because it fails to meet this second criterion, namely, consistency. T-61 does not act consistently: it can cause animals intense pain soon after initial administration, or even a curare-like paralysis of respiration before the animal is unconscious.

If T-61 were consistent in its pharmacological effects and caused death swiftly and without pain or distress to animals, then veterinarians would be consistent in their recognition and acceptance of T-61 as a humane compound for euthanasia. But the truth is that there is no unanimous consensus in the veterinary profession to this end as evidenced by Andrew Rowan's survey.

That only 27.8 percent of respondents approved of T-61 and 44 percent experienced bad reactions in animals to this compound is clear evidence that T-61 does not meet the humane euthanasia criterion of consistency or reliability.

Bad reactions reported by veterinarians in Rowan's study included: muscle tremors and excitation, including convulsions, crying, and agonal thrashing; and prolonged heart activity, the heart beating for five minutes or longer after brain death had apparently occurred.

The manufacturers will claim that such undesirable reactions occur because the veterinarians have not followed their printed instructions for proper administration of T-61. But surely so many veterinarians who are opposed to the use of T-61 in Rowan's survey cannot be that illiterate and incompetent as to be incapable of administering T-61 properly.

Furthermore, since the manufacturers clearly admit that improper administration can result in inhumane side-effects on animals, it is self-evident that since competent and experienced veterinarians have had problems with this compound, animal shelter and animal control personnel using T-61 are likely to encounter problems even more frequently.

Thus T-61 should not be approved for use by laypersons in animal shelters and it is for these reasons that The Humane Society of the United States (HSUS) has concluded in a recent publication that " $T-61$ is an impractical and unacceptable euthanasia drug. It is not a controlled substance, but it is one of the more expensive euthanasia drugs." This publication also emphasizes that T-61 is of limited use because "a major limitation... is that it should be injected only intravenously and not administered by any other route; an additional limitation is that it should be administered at a precise rate. Not only does the skill of the 
euthanasia technician vary from person to person, but the reactions of the animals are variable and unpredictable."

This publication approves of sodium pentobarbital as the best euthanasia method available and endorses the use of FP-3 (sodium pentobarbital plus lidocaine), the added benefit being that this is easier to obtain as a Schedule III drug rather than Schedule II as sodium pentobarbital is classified.

Further evidence in support of T-61 being rejected as being an unsatisfactory and unreliable euthanasia compound comes from the United Kingdom, where T-61 is not accepted by the veterinary profession. British veterinarian Deborah J. Baker, employed by Hoechst Company, manufacturers of T-61 states, in a letter to The HSUS (7 August 1980):

"...for the last four years, this product has been unavailable in the U.K. It was withdrawn because although for Euthanasia the mode of action seems good, in practice, I gather that animals euthanized with this tended to have rather distressing, painful convulsions accompanied by howling prior to their demise, this being because the phases, i.e. induction stage, anaesthetic stage and respiratory paralysis do not occur in that order, and one tends to get respiratory paralysis prior to anaesthesia being completed. I, myself, therefore, would not recommend its use for euthanasia purposes."

A study by Dr. William Lumb comparing T-61 and sodium pentobarbital published in the Journal of the American Veterinary Medical Association comparing the effects of sodium pentobarbital and T-61 (January 15, 1968) is often cited as showing that T-61 is superior because some of the dogs given sodium pentobarbital did not die. While it might be unfair to suggest that this study, funded by the manufacturers of T-61, was rigged to ensure a favorable outcome for them, it is obvious that the investigator avoided overdosing the dogs with sodium pentobarbital in order to ensure that they would not recover, which is common practice when using this drug for euthanasia. Dr. Lumb used a weaker (anaesthetic-surgery) strength solution of sodium pentobarbital in this study, than is routinely used when this drug is employed for euthanasia. Little wonder therefore that some dogs recovered.

It is obvious from the above evidence, and in view of the fact that there are more humane alternatives to using T-61 as a euthanasia agent, that T-61 should not be approved for euthanasia purposes in animal shelters. Nor should it be recognized as an acceptable agent of euthanasia. 